\title{
Continuous knapsack sets with divisible capacities
}

\author{
Laurence A. Wolsey • Hande Yaman
}

Received: 22 November 2013 / Accepted: 31 January 2015 / Published online: 17 February 2015

(C) Springer-Verlag Berlin Heidelberg and Mathematical Optimization Society 2015

\begin{abstract}
We study two continuous knapsack sets $\mathcal{Y}_{\geq}$and $\mathcal{Y}_{\leq}$with $n$ integer, one unbounded continuous and $m$ bounded continuous variables in either $\geq$ or $\leq$ form. When the coefficients of the integer variables are integer and divisible, we show in both cases that the convex hull is the intersection of the bound constraints and $2^{m}$ polyhedra arising as the convex hulls of continuous knapsack sets with a single unbounded continuous variable. The latter convex hulls are completely described by an exponential family of partition inequalities and a polynomial size extended formulation is known in the $\geq$ case. We also provide an extended formulation for the $\leq$ case. It follows that, given a specific objective function, optimization over both $\mathcal{Y}_{\geq}$and $\mathcal{Y}_{\leq}$can be carried out by solving $m$ polynomial size linear programs. A further consequence of these results is that the coefficients of the continuous variables all take the values 0 or 1 (after scaling) in any non-trivial facet-defining inequality of the convex hull of such sets.
\end{abstract}

Keywords Continuous knapsack set - Splittable flow arc set - Divisible capacities · Partition inequalities · Convex hull

Mathematics Subject Classification $90 \mathrm{C} 11 \cdot 90 \mathrm{C} 27$

\footnotetext{
L. A. Wolsey

CORE, Université Catholique de Louvain, Voie du Roman Pays 34, 1348 Louvain-la-Neuve, Belgium e-mail: laurence.wolsey@uclouvain.be
}

H. Yaman $(\varangle)$

Department of Industrial Engineering, Bilkent University, 06800 Bilkent, Ankara, Turkey

e-mail: hyaman@bilkent.edu.tr 


\section{Introduction}

Let $m$ and $n$ be positive integers, $M=\{1, \ldots, m\}, M_{0}=M \cup\{0\}$ and $N=\{1, \ldots, n\}$. The parameters $a_{i}$ for $i \in M, c_{1} \leq \ldots \leq c_{n}$ and $b$ are positive integers. The $\left\{c_{j}\right\}$ are distinct. The multi-item continuous $\geq$-knapsack set is

$$
\mathcal{Y}_{\geq}=\left\{(y, x) \in \mathbb{Z}_{+}^{n} \times \mathbb{R}_{+}^{m+1}: \sum_{j \in N} c_{j} y_{j}+\sum_{i \in M_{0}} x_{i} \geq b, x_{i} \leq a_{i}, i \in M\right\},
$$

the multi-item continuous $\leq$-knapsack set is

$$
\mathcal{Y}_{\leq}=\left\{(y, x) \in \mathbb{Z}_{+}^{n} \times \mathbb{R}_{+}^{m+1}: \sum_{j \in N} c_{j} y_{j} \leq b+\sum_{i \in M_{0}} x_{i}, x_{i} \leq a_{i}, i \in M\right\}
$$

and the unbounded single item continuous knapsack sets are

$$
\mathcal{Q}_{\geq}=\left\{(y, x) \in \mathbb{Z}_{+}^{n} \times \mathbb{R}_{+}^{1}: \sum_{j \in N} c_{j} y_{j}+x \geq b\right\}
$$

and

$$
\mathcal{Q}_{\leq}=\left\{(y, x) \in \mathbb{Z}_{+}^{n} \times \mathbb{R}_{+}^{1}: \sum_{j \in N} c_{j} y_{j} \leq b+x\right\}
$$

These sets arise as relaxations of many mixed-integer programming problems and consequently strong valid inequalities for these sets can be used in solving more complicated problems. Indeed, many strong inequalities used in the literature can be obtained using such knapsack relaxations. For books and inequalities on general knapsack sets, see among others $[2,6,12,14,17]$.

Here we consider a case in which there is special structure, specifically the coefficients of the integer variables are divisible $1\left|c_{1}\right| \cdots \mid c_{n}$. Generalizing results of Pochet and Wolsey [16] for the $\geq$-knapsack set, we show that $\mathcal{Q}_{\geq}$and $\mathcal{Q}_{\leq}$can be described by two closely related families of "partition" inequalities. This in turn leads to complete polyhedral descriptions of $\mathcal{Y}_{\geq}$and $\mathcal{Y}_{\leq}$. Specifically we show that

$$
\operatorname{conv}\left(\mathcal{Y}_{\geq}\right)=\cap_{S \subseteq M} \operatorname{conv}\left(\mathcal{Q}_{\geq}^{S}\right) \cap\left\{(y, x): x_{i} \leq a_{i}, i \in M\right\}
$$

where

$$
\mathcal{Q}_{\geq}^{S}=\left\{(y, x) \in \mathbb{Z}_{+}^{n} \times \mathbb{R}^{m+1}: \sum_{j \in N} c_{j} y_{j}+x(S \cup\{0\}) \geq b-a(M \backslash S), x(S \cup\{0\}) \geq 0\right\}
$$

with a similar result for $\mathcal{Y}_{\leq}\left(\right.$where $v(A)=\sum_{a \in A} v_{a}$ for a vector $v$ and a set $\left.A\right)$. 
For $\mathcal{Y}_{\geq}$, this generalizes a result of Magnanti et al. [10] concerning the "single arc flow set" in which they show (modulo complementation of the continuous variables) that when $n=1$, the convex hull of $\mathcal{Y}_{\geq} \cap\left\{(y, x): x_{0}=0\right\}$ is completely described by adding the "residual capacity" or mixed integer rounding (MIR) inequalities one for each of the relaxations

$$
c_{1} y_{1}+x(S) \geq b-a(M \backslash S), x(S) \geq 0, y \in \mathbb{Z}_{+}^{1},
$$

where $S \subseteq M$. Atamtürk and Rajan [3] give a polynomial time separation algorithm for the residual capacity inequalities. Magnanti et al. [11] generalize the residual capacity inequalities for the two facility splittable flow arc set when $n=2, c_{1}=1$ and state without proof that addition of the two MIR inequalities arising for each choice of $S \subseteq M$ suffices to give the convex hull.

Other work on divisible knapsack sets includes a convex hull description of the integer $\leq$-knapsack set $\left\{y \in \mathbb{Z}_{+}^{n}: \sum_{j \in N} c_{j} y_{j} \leq b\right\}$ consisting of $n$ Chvatal-Gomory rounding inequalities by Marcotte [13] and a study of Pochet and Weismantel [15] of the case with bounded variables. Other (continuous) knapsack sets with special structure whose polyhedral structure has been studied include the set $\mathcal{Q}_{\leq}$with $n=2$ and $c_{1}, c_{2}$ arbitrary positive integers (Agra and Constantino [1] and Dash et al. [4]), as well as 0-1 knapsack sets with super-increasing coefficients (Laurent and Sassano [8]) and more recently a generalization with bounded integer variables (Gupte [5]).

The rest of the paper is organized as follows. In Sect. 2, we review some results on knapsack sets with divisible capacities. In Sect. 3 we study the convex hull of the multi-item continuous $\geq$-knapsack set and prove that the original constraints and the so-called "partition inequalities" are sufficient to describe the convex hull when the capacities are divisible. A result on the convex hull of the two-sided integer knapsack $\left\{y \in \mathbb{Z}_{+}^{n}: b^{\prime} \leq \sum_{j \in N} c_{j} y_{j} \leq b\right\}$ is an immediate corollary. In Sect. 4 we show that a new, but related, family of partition inequalities is valid for the continuous $\leq$-knapsack set with one unbounded continuous variable $\mathcal{Q}_{\leq}$. We also give a polynomial size extended formulation for $\mathcal{Q}_{\leq}$. In Sect. 5 we provide a convex hull description for the case of $m$ bounded continuous variables and one unbounded continuous variable $\mathcal{Y}_{\leq}$. We conclude in Sect. 6.

\section{The $\geq-$-knapsack set and partition inequalities}

Throughout the paper, we assume that the capacities are divisible. We use the notation $c y=\sum_{j \in N} c_{j} y_{j}$. Below we present results from Pochet and Wolsey [16] that will be used in Sect. 3.

Consider the integer $\geq$-knapsack set

$$
\mathcal{C}=\left\{y \in \mathbb{Z}_{+}^{n}: c y \geq b\right\}
$$

with $c_{1}=1$. Let $v(b)$ be the index with $c_{v(b)} \leq b<c_{v(b)+1}$ if such an index exists and be $n$ otherwise. 
For some integer $p \geq 1$, let $\left\{i_{1}=1, i_{1}+1, \ldots, j_{1}\right\},\left\{i_{2}=j_{1}+1, i_{2}+1, \ldots, j_{2}\right\}$ $, \ldots,\left\{i_{p}=j_{p-1}+1, i_{p}+1, \ldots, j_{p}=n\right\}$ be a partition of $\{1, \ldots, n\}$ such that $i_{p} \leq$ $v(b)$ and $i_{t}=j_{t-1}+1$ for $t=2, \ldots, p$. Compute

$$
\beta_{p}=b, \kappa_{t}=\left\lceil\frac{\beta_{t}}{c_{i_{t}}}\right\rceil, \mu_{t}=\left(\kappa_{t}-1\right) c_{i_{t}} \text { and } \beta_{t-1}=\beta_{t}-\mu_{t} \text { for } t=p, \ldots, 1 .
$$

Note that the $\beta_{t}$ are positive and nondecreasing in $t$ and $\kappa_{t} \geq 1$ for all $t$.

The partition inequality is

$$
\sum_{t=1}^{p} \prod_{l=1}^{t-1} \kappa_{l} \sum_{j=i_{t}}^{j_{t}} \min \left\{\frac{c_{j}}{c_{i_{t}}}, \kappa_{t}\right\} y_{j} \geq \prod_{t=1}^{p} \kappa_{t}
$$

Pochet and Wolsey [16] prove that these inequalities are valid for the integer $\geq$ knapsack set $\mathcal{C}$. They also prove the following result on $\operatorname{conv}(\mathcal{C})$ :

Theorem 1 The convex hull of $\mathcal{C}$ is described by its constraints and the partition inequalities (1).

In addition, Pochet and Wolsey [16] establish the following results.

Theorem 2 Let $g \in \mathbb{R}^{n}$ and $\left\{i_{1}, \ldots, j_{1}\right\},\left\{i_{2}, \ldots, j_{2}\right\}, \ldots,\left\{i_{p}, \ldots, j_{p}\right\}$ be a partition of $\{1, \ldots, n\}$ such that $i_{p} \leq v(b)$. Suppose that $g>0, \frac{g_{j}}{c_{j}}$ is constantfor $j=i_{t}, \ldots, j_{t}$ and $\frac{g_{i_{t}}}{c_{i_{t}}}>\frac{g_{i_{t+1}}}{c_{i_{t+1}}}$ for $t=1, \ldots, p-1, \frac{g_{i p}}{c_{i_{p}}}=\frac{g_{j}}{c_{j}}$ for $j=i_{p}, \ldots, v(b)$ and $g_{v(b)+1}=g_{j}$ for $j=v(b)+1, \ldots, n$.

i. All optimal solutions of $\min \left\{\sum_{j=1}^{n} g_{j} y_{j}: c y \geq b, y \in \mathbb{Z}_{+}^{n}\right\}$ satisfy (1) at equality.

ii. All optimal solutions of $\min \left\{\sum_{j=i_{2}}^{n} g_{j} y_{j}: \sum_{j=i_{2}}^{n} c_{j} y_{j} \geq\left\lceil\frac{b}{c_{i_{2}}}\right\rceil c_{i_{2}}, y \in \mathbb{Z}_{+}^{n}\right\}$ satisfy

$$
\sum_{t=2}^{p} \prod_{l=2}^{t-1} \kappa_{l} \sum_{j=i_{t}}^{j_{t}} \min \left\{\frac{c_{j}}{c_{i_{t}}}, \kappa_{t}\right\} y_{j}=\prod_{t=2}^{p} \kappa_{t}
$$

iii. All optimal solutions of $\min \left\{\sum_{j=i_{2}}^{n} g_{j} y_{j}: \sum_{j=i_{2}}^{n} c_{j} y_{j}=\left\lfloor\frac{b}{c_{i_{2}}}\right\rfloor c_{i_{2}}, y \in \mathbb{Z}_{+}^{n}\right\}$ satisfy

$$
\sum_{t=2}^{p} \prod_{l=2}^{t-1} \kappa_{l} \sum_{j=i_{t}}^{j_{t}} \min \left\{\frac{c_{j}}{c_{i_{t}}}, \kappa_{t}\right\} y_{j}=\prod_{t=2}^{p} \kappa_{t}-1
$$

\section{The multi-item continuous $\geq-$-knapsack set}

In this section, we study the convex hull of the multi-item continuous $\geq$-knapsack set $\mathcal{Y}_{\geq}$when the capacities are divisible.

Our goal now is to show that

$$
\operatorname{conv}\left(\mathcal{Y}_{\geq}\right)=\cap_{S \subseteq M} \operatorname{conv}\left(\mathcal{Q}_{\geq}^{S}\right) \cap\left\{(y, x): x_{i} \leq a_{i}, i \in M\right\}
$$


where

$$
\mathcal{Q}_{\geq}^{S}=\left\{(y, x) \in \mathbb{Z}_{+}^{n} \times \mathbb{R}^{m+1}: x\left(S_{0}\right)+c y \geq b-a(M \backslash S), x\left(S_{0}\right) \geq 0\right\},
$$

and $S_{0}=S \cup\{0\}$.

Note that in the extreme points of $\operatorname{conv}\left(\mathcal{Y}_{\geq}\right), x_{0}$ takes integer values. We give the convex hull proof for $\mathcal{Y}_{\geq}^{\prime}=\mathcal{Y}_{\geq} \cap\left\{(y, x): x_{0}=0\right\}$ since $x_{0}$ can be considered an integer variable with coefficient 1 .

Given $S \subseteq M$, consider the following reformulation of $\mathcal{Q}_{\geq}^{S}$

$$
\left\{\left(y_{0}, y, x\right) \in \mathbb{R}_{+}^{1} \times \mathbb{Z}_{+}^{n} \times \mathbb{R}^{m+1}: y_{0}+c y \geq b-a(M \backslash S), y_{0}=x\left(S_{0}\right)\right\} .
$$

As the data is integral, $y_{0}$ takes an integer value in every extreme point of the convex hull of the above set. Setting $y_{0}$ integer, we obtain the divisible capacity knapsack cover set

$$
\left\{\left(y_{0}, y, x\right) \in \mathbb{Z}_{+}^{n+1} \times \mathbb{R}^{m+1}: \sum_{j \in N_{0}} c_{j} y_{j} \geq B(S), y_{0}=x\left(S_{0}\right)\right\}
$$

where $N_{0}=N \cup\{0\}, c_{0}=1$ and $B(S)=b-a(M \backslash S)$.

Proposition 1 Let $S \subseteq M,\left\{i_{1}=0, \ldots, j_{1}\right\}, \ldots,\left\{i_{p}, \ldots, j_{p}=n\right\}$ be a partition of $\{0,1, \ldots, n\}$ such that $i_{p} \leq v(B(S))$ and $i_{t}=j_{t-1}+1$ for $t=2, \ldots, p$. Let $\beta_{p}=B(S), \kappa_{t}=\left\lceil\frac{\beta_{t}}{c_{i_{t}}}\right\rceil, \mu_{t}=\left(\kappa_{t}-1\right) c_{i_{t}}$ and $\beta_{t-1}=\beta_{t}-\mu_{t}$ for $t=p, \ldots, 1$. Then the partition inequality

$$
x\left(S_{0}\right)+\sum_{j=1}^{j_{1}} \min \left\{c_{j}, \kappa_{1}\right\} y_{j}+\sum_{t=2}^{p} \prod_{l=1}^{t-1} \kappa_{l} \sum_{j=i_{t}}^{j_{t}} \min \left\{\frac{c_{j}}{c_{i_{t}}}, \kappa_{t}\right\} y_{j} \geq \prod_{t=1}^{p} \kappa_{t}
$$

is valid for both $\mathcal{Q}_{\geq}^{S}$ and $\mathcal{Y}_{\geq}$.

Using Theorem 1, we obtain the following result:

Corollary 1 conv $\left(\mathcal{Q}_{\geq}\right)$is described by its constraints and partition inequalities (2) with $x=x\left(S_{0}\right)$.

Theorem 3 conv $\left(\mathcal{Y}_{\geq}^{\prime}\right)$ is described by the initial constraints and the partition inequalities (2).

Proof We use the technique of Lovász [9]. Suppose that we minimize $\sum_{i \in M} h_{i} x_{i}+$ $\sum_{j \in N} g_{j} y_{j}$ over $\mathcal{Y}_{\geq}^{\prime}$ where $(h, g) \neq 0$. We need $g \geq 0$ for the problem to be bounded. Let $\Omega(h, g)$ be the set of optimal solutions.

If $h_{i}<0$ for some $i \in M$, then $\Omega(h, g) \subseteq\left\{(y, x): x_{i}=a_{i}\right\}$. If $h \gtrless 0$ and $g=0$ with $h_{i}>0$ for some $i \in M, \Omega(h, g) \subseteq\left\{(y, x): x_{i}=0\right\}$. If $g_{j}=0$ and $g_{j^{\prime}}>0$ for some pair $j, j^{\prime} \in N, \Omega(h, g) \subseteq\left\{(y, x): y_{j^{\prime}}=0\right\}$. Thus we are left with $h \geq 0$ and 
$g>0$. Let $S=\left\{i \in M: h_{i}>0\right\}$. If $B(S) \leq 0$, then $\Omega(h, g) \subseteq\left\{(y, x): y_{j}=0\right\}$ for all $j \in N$.

Now suppose that $B(S)>0$. If there exist indices $j_{1}<j_{2} \leq v(B(S))$ such that $\frac{c_{j_{2}}}{c_{j_{1}}} g_{j_{1}}<g_{j_{2}}$, then $y_{j_{2}}=0$ for all $(y, x) \in \Omega(h, g)$. Similarly, if there exist $j_{1}$ and $j_{2}$ in $\{v(B(S))+1, \ldots, n\}$ such that $g_{j_{1}}<g_{j_{2}}$, then $y_{j_{2}}=0$ for all $(y, x) \in \Omega(h, g)$.

We are left with $B(S)>0, \frac{g_{1}}{c_{1}} \geq \ldots \geq \frac{g_{v(B(S))}}{c_{v(B(S))}}>0$ and $g_{v(B(S))+1}=\ldots=g_{n}>0$. One possibility is that $\Omega(h, g) \subseteq\{(y, x): x(M)+c y=b\}$.

The last case to be considered is that in which there exists an optimal solution $\left(y^{*}, x^{*}\right)$ with $x^{*}(M)+c y^{*}>b$. Note that $y^{*} \neq 0$ for any such solution since $B(S)>0$. Let $q$ be the smallest index such that there exists an optimal solution $\left(y^{*}, x^{*}\right)$ with $x^{*}(M)+c y^{*}>b$ and $y_{q}^{*}>0$. Then we know the following:

a. $x_{i}=0$ for all $i \in S$ in any optimal solution $(y, x)$ with $x(M)+c y>b$. Otherwise one can decrease some $x_{i}$ with $i \in S$ by a small amount, remain valid and improve the objective function.

b. $\frac{g_{q-1}}{c_{q-1}}>\frac{g_{q}}{c_{q}}$. For $j \in N$, define $e_{j}$ to be the $j$-th unit vector of size $n$. If $\frac{g_{q-1}}{c_{q-1}}=\frac{g_{q}}{c_{q}}$, then $\left(x^{*}, y^{*}-e_{q}+\frac{c_{q}}{c_{q-1}} e_{q-1}\right)$ is also optimal, contradicting the definition of $q$.

c. $c_{q} \backslash B(S)$. Suppose on the contrary that $c_{q}$ divides $B(S)$. Let $\left(y^{*}, x^{*}\right)$ be an optimal solution with $x^{*}(M)+c y^{*}>b$ and $y_{q}^{*}>0$. We have $\sum_{j=q}^{n} c_{j} y_{j}^{*}>B(S)$ since $y_{j}^{*}=0$ for $j=1, \ldots, q-1, x^{*}(S)=0$ and $x^{*}(M)+c y^{*}>b$. As $\sum_{j=q}^{n} c_{j} y_{j}^{*}$ is a multiple of $c_{q}$, it follows that $\sum_{j=q}^{n} c_{j} y_{j}^{*} \geq B(S)+c_{q}$. But now $\left(x^{*}, y^{*}-e_{q}\right)$ is feasible and cheaper since $g_{q}>0$, contradicting the optimality of $\left(x^{*}, y^{*}\right)$.

d. $\quad c y \geq\left\lfloor\frac{B(S)}{c_{q}}\right\rfloor c_{q}$ in any optimal solution $(y, x)$. If $S=\emptyset$, then by feasibility we require $c y \geq B(S)$ and hence $c y \geq\left\lfloor\frac{B(S)}{c_{q}}\right\rfloor c_{q}$. If $S \neq \emptyset$, define $\phi(\sigma)=$ $\min \left\{\sum_{i \in S} h_{i} x_{i}: x(S) \geq B(S)-\sigma, 0 \leq x_{i} \leq a_{i} i \in S\right\}$. Optimality of $\left(y^{*}, x^{*}\right)$ as in c) above implies $g_{q} \leq \phi\left(c\left(y^{*}-e_{q}\right)\right)-\phi\left(c y^{*}\right)$. The fact that the knapsack cover constraint is not tight together with $c_{q}$ does not divide $B(S)$ implies that $c y^{*} \geq\left\lfloor\frac{B(S)}{c_{q}}\right\rfloor c_{q}+c_{q}$. Suppose now that $\left(y^{\prime}, x^{\prime}\right)$ is an optimal solution with $c y^{\prime}<\left\lfloor\frac{B(S)}{c_{q}}\right\rfloor c_{q}$. Now $\phi$ is a piecewise linear convex function with $\phi(\sigma)>0$ for $\sigma<B(S)$ and $\phi(\sigma)=0$ for $\sigma \geq B(S)$. It is strictly decreasing on the interval $[\max (0, B(S)-a(S)), B(S)]$. Therefore, as $c y^{\prime} \geq B(S)-a(S), c y^{\prime}<$ $c\left(y^{*}-e_{q}\right)<B(S)$ and $c y^{*}>B(S)$, one has $\phi\left(c y^{\prime}\right)-\phi\left(c\left(y^{\prime}+e_{q}\right)\right)>\phi\left(c\left(y^{*}-\right.\right.$ $\left.\left.e_{q}\right)\right)-\phi\left(c y^{*}\right)$. It follows that $\phi\left(c y^{\prime}\right)-\phi\left(c\left(y^{\prime}+e_{q}\right)\right)>g_{q}$ and thus $g\left(y^{\prime}+\right.$ $\left.e_{q}\right)+\phi\left(c\left(y^{\prime}+e_{q}\right)\right)<g y^{\prime}+\phi\left(c y^{\prime}\right)$. So increasing $y_{q}^{\prime}$ by 1 and picking the best $x$ improves the objective function value. Hence $\left(y^{\prime}, x^{\prime}\right)$ cannot be optimal.

Now let $y_{0}=x(S)$ and $q$ be as defined above. Let $\left\{i_{1}, \ldots, j_{1}\right\},\left\{i_{2}, \ldots, j_{2}\right\}$, $\ldots,\left\{i_{p}, \ldots, j_{p}\right\}$ be a partition of $\{0, \ldots, n\}$ such that $i_{1}=0, j_{1}=q-1$, $i_{p} \leq v(B(S)), \frac{g_{j}}{c_{j}}$ is constant for $j=i_{t}, \ldots, j_{t}$ and $\frac{g_{i_{t}}}{c_{i_{t}}}>\frac{g_{i_{t+1}}}{c_{i_{t+1}}}$ for $t=2, \ldots, p-1$, $\frac{g_{i_{p}}}{c_{i_{p}}}=\frac{g_{j}}{c_{j}}$ for $j=i_{p}, \ldots, v(B(S))$ and $g_{v(B(S))+1}=g_{j}$ for $j=v(B(S))+1, \ldots, n$. We claim that all optimal solutions satisfy the corresponding partition inequality (2) at equality. 
Take an arbitrary point $(y, x) \in \Omega(h, g)$. If the knapsack cover constraint is not tight at $(y, x)$, then $x_{i}=0$ for all $i \in S, y_{j}=0$ for $j=1, \ldots, q-1$ and $\left(y_{q}, \ldots, y_{n}\right)$ is optimal for the problem of minimizing $\sum_{j=q}^{n} g_{j} \tilde{y}_{j}$ subject to $\sum_{j=q}^{n} c_{j} \tilde{y}_{j} \geq\left\lceil\frac{B(S)}{c_{q}}\right\rceil c_{q}$ and $\tilde{y} \in \mathbb{Z}_{+}^{n}$. Then $\sum_{t=2}^{p} \prod_{l=2}^{t-1} \kappa_{l} \sum_{j=i_{t}}^{j_{t}} \min \left\{\frac{c_{j}}{c_{i_{t}}}, \kappa_{t}\right\} y_{j}=\prod_{t=2}^{p} \kappa_{t}$ using (ii) of Theorem 2. Multiplying by $\kappa_{1} \geq 1$ and adding the terms $x(S)+\sum_{j=0}^{j_{1}} \min \left(c_{j}, \kappa_{1}\right) y_{j}=$ 0 , we see that $(y, x)$ satisfies $(2)$ at equality.

Now suppose that the knapsack cover constraint is tight at $(y, x)$. There are two cases.

Case 1) $x(M \backslash S)<a(M \backslash S)$. Then $x_{i}=0$ for all $i \in S$ since otherwise one can decrease some $x_{i}$ with $i \in S$ and increase some $x_{i^{\prime}}$ with $i^{\prime} \in M \backslash S$ by the same amount and improve the objective function value. Now the set of optimal points have the same $y$ values as in the above case, completed by $x_{i}=0$ for $i \in S$ and $x_{i}$ for $i \in M \backslash S$ satisfying $0 \leq x_{i} \leq a_{i}, x(M \backslash S)=b-c y$.

Case 2) $x_{i}=a_{i}$ for $i \in M \backslash S$. Since (from (c)) $B(S)$ is not a multiple of $c_{q}$, we have $\sum_{j=q}^{n} c_{j} y_{j} \leq\left\lfloor\frac{B(S)}{c_{q}}\right\rfloor c_{q}$. From (b), we know that $\frac{g_{q-1}}{c_{q-1}}>\frac{g_{q}}{c_{q}}$ and from (d) we know that $\sum_{j=1}^{n} c_{j} y_{j} \geq\left\lfloor\frac{B(S)}{c_{q}}\right\rfloor c_{q}$. Hence $\sum_{j=q}^{n} c_{j} y_{j}=\left\lfloor\frac{B(S)}{c_{q}}\right\rfloor c_{q}$. Since the knapsack constraint is tight, we have $y_{0}+\sum_{j=1}^{q-1} c_{j} y_{j}=B(S)-\left[\frac{B(S)}{c_{q}}\right] c_{q}$. As $B(S)-\left\lfloor\frac{B(S)}{c_{q}}\right\rfloor c_{q}=\kappa_{1},(y, x)$ satisfies $y_{0}+\sum_{j=1}^{q-1} \min \left\{c_{j}, \kappa_{1}\right\} y_{j}=\kappa_{1}$. Using (iii) of Theorem 2, any optimal solution to the problem of minimizing $\sum_{j=q}^{n} g_{j} y_{j}$ subject to $\sum_{j=q}^{n} c_{j} y_{j}=\left\lfloor\frac{B(S)}{c_{q}}\right\rfloor c_{q}$ and $y \in \mathbb{Z}_{+}^{n}$ satisfies $\sum_{t=2}^{p} \prod_{l=2}^{t-1} \kappa_{l} \sum_{j=i_{t}}^{j_{t}} \min \left\{\frac{c_{j}}{c_{i_{t}}}, \kappa_{t}\right\} y_{j}=\prod_{t=2}^{p} \kappa_{t}-1$. Now

$$
\begin{aligned}
& \sum_{t=1}^{p} \prod_{l=1}^{t-1} \kappa_{l} \sum_{j=i_{t}}^{j_{t}} \min \left\{\frac{c_{j}}{c_{i_{t}}}, \kappa_{t}\right\} y_{j} \\
& =x(S)+\sum_{j=1}^{q-1} \min \left\{c_{j}, \kappa_{1}\right\} y_{j}+\sum_{t=2}^{p} \prod_{l=1}^{t-1} \kappa_{l} \sum_{j=i_{t}}^{j_{t}} \min \left\{\frac{c_{j}}{c_{i_{t}}}, \kappa_{t}\right\} y_{j} \\
& =x(S)+\sum_{j=1}^{q-1} \min \left\{c_{j}, \kappa_{1}\right\} y_{j}+\kappa_{1}\left(\sum_{t=2}^{p} \prod_{l=2}^{t-1} \kappa_{l} \sum_{j=i_{t}}^{j_{t}} \min \left\{\frac{c_{j}}{c_{i_{t}}}, \kappa_{t}\right\} y_{j}\right) \\
& =\kappa_{1}+\kappa_{1}\left(\prod_{t=2}^{p} \kappa_{t}-1\right)=\prod_{t=1}^{p} \kappa_{t} .
\end{aligned}
$$

Thus all optimal solutions satisfy this partition inequality (2) at equality.

As $\operatorname{conv}\left(\mathcal{Q}_{\geq}^{S}\right)$ is described by the trivial inequalities and the partition inequalities, we get $\operatorname{conv}\left(\overline{\mathcal{Y}}_{\geq}\right)=\cap_{S \subseteq M} \operatorname{conv}\left(\mathcal{Q}_{\geq}^{S}\right) \cap\left\{(y, x): x_{i} \leq a_{i}, i \in M\right\}$.

As a corollary, one obtains a simple result concerning the intersection of two parallel divisible knapsack sets. 
Theorem 4 conv $\left(\left\{y \in \mathbb{Z}_{+}^{n}: b-a \leq c y \leq b\right\}\right)=\operatorname{conv}\left(\left\{y \in \mathbb{Z}_{+}^{n}: b-a \leq c y\right\}\right) \cap$ conv $\left(\left\{y \in \mathbb{Z}_{+}^{n}: c y \leq b\right\}\right)$.

Proof Consider the case of Theorem 3 when $m=1$, i.e., when there is a single continuous variable. Then we have

$$
\begin{aligned}
\operatorname{conv}\left(\mathcal{Y}_{\geq}^{\prime}\right)= & \operatorname{conv}\left(\left\{(y, x) \in \mathbb{Z}_{+}^{n} \times \mathbb{R}: c y \geq b-a\right\}\right) \\
& \cap \operatorname{conv}\left(\left\{(y, x) \in \mathbb{Z}_{+}^{n} \times \mathbb{R}_{+}: x+c y \geq b\right\}\right) \cap\left\{(y, x) \in \mathbb{R}^{n+1}: x \leq a\right\} .
\end{aligned}
$$

We use the fact that if the inequality $x+c y \geq b$ defines a face of $\operatorname{conv}(Z)$, then

$$
\operatorname{conv}(Z) \cap\{(y, x): x+c y=b\}=\operatorname{conv}(Z \cap\{x=b-c y\}) .
$$

We now intersect the above sets with the hyperplane $\{(y, x): x+c y=b\}$ and then project into the space of the $y$ variables.

Intersecting the set on the left gives:

$$
\begin{aligned}
\operatorname{conv} & \left(\left\{(y, x) \in \mathbb{Z}_{+}^{n} \times \mathbb{R}: x+c y \geq b, 0 \leq x \leq a\right\}\right) \cap\{(y, x): x+c y=b\} \\
= & \operatorname{conv}\left(\left\{(y, x) \in \mathbb{Z}_{+}^{n} \times \mathbb{R}: 0 \leq x=b-c y \leq a\right\}\right) \\
= & \operatorname{conv}\left(\left\{(y, x) \in \mathbb{Z}_{+}^{n} \times \mathbb{R}: b-a \leq c y \leq b, x=b-c y\right\}\right) \\
= & \operatorname{conv}\left(\left\{(y, x) \in \mathbb{Z}_{+}^{n} \times \mathbb{R}: b-a \leq c y \leq b\right\}\right) \cap\{(y, x): x=b-c y\},
\end{aligned}
$$

and the projection is conv $\left(\left\{y \in \mathbb{Z}_{+}^{n}: b-a \leq c y \leq b\right\}\right)$.

Using the facial property, we can show that the intersection of the set on the right hand side with the set $\{(y, x): x=b-c y\}$ is equal to conv $\left(\left\{(y, x) \in \mathbb{Z}_{+}^{n} \times \mathbb{R}: c y\right.\right.$ $\geq b-a, x=b-c y\}) \cap \operatorname{conv}\left(\left\{(y, x) \in \mathbb{Z}_{+}^{n} \times \mathbb{R}: c y \leq b, x=b-c y\right\}\right) \cap\{(y, x)$ $\left.\in \mathbb{R}^{n} \times \mathbb{R}: b-c y \leq a, x=b-c y\right\}$. The projection of the first gives directly conv $\left(\left\{y \in \mathbb{Z}_{+}^{n}: c y \geq b-a\right\}\right)$, the second conv $\left(\left\{y \in \mathbb{Z}_{+}^{n}: c y \leq b\right\}\right)$ and the third $\{y: c y \geq b-a\}$. The claim follows.

\section{The continuous $\leq-$ knapsack set}

Now we study the convex hull of the continuous $\leq$-knapsack set, namely the set

$$
\mathcal{Q}_{\leq}=\left\{(y, x) \in \mathbb{Z}_{+}^{n} \times \mathbb{R}_{+}^{1}: \sum_{j=1}^{n} c_{j} y_{j} \leq b+x\right\},
$$

where again the data are integer and $c_{1}|\cdots| c_{n}$. Initially we suppose that $c_{1}$ and hence $c_{i}$ for all $i$ do not divide $b$. Below we will define a new family of " $\leq$-partition" inequalities.

Given a partition $\left\{i_{1}, \ldots, j_{1}\right\}, \cdots,\left\{i_{p}, \ldots, j_{p}\right\}$ of $\{1, \ldots, n\}$ into intervals, let $\beta_{p}=b, \kappa_{t}=\left\lceil\frac{\beta_{t}}{c_{i_{t}}}\right\rceil, \mu_{t}=\left(\kappa_{t}-1\right) c_{i_{t}}, \beta_{t-1}=\beta_{t}-\mu_{t}$ and $s_{t}=c_{i_{t}}-\beta_{t-1}$ for $t=p, \ldots, 1$.

We first consider the partition into singletons $(\{1\}, \cdots,\{n\})$. 
Observation 1 The following $n+1$ points

$$
\begin{aligned}
z^{k} & =\left(y^{k}, x^{k}\right)=\left(0,0, \ldots, 0, \kappa_{k}, \kappa_{k+1}-1 \ldots, \kappa_{n}-1, s_{k}\right) k=1, \ldots, n \\
z^{0} & =\left(y^{0}, x^{0}\right)=\left(\kappa_{1}-1, \ldots, \kappa_{k}-1, \kappa_{k+1}-1 \ldots, \kappa_{n}-1,0\right)
\end{aligned}
$$

are in $\mathcal{Q}_{\leq}$.

Proof The point $z^{n}$ is in $\mathcal{Q}_{<}$since $c y^{n}-x^{n}=c_{n} \kappa_{n}-s_{n}=c_{n} \kappa_{n}-\left(c_{n}-b+c_{n}\left(\kappa_{n}-\right.\right.$ $1))=b$. For $1 \leq k \leq n-1,(c,-1)\left(z^{k+1}-z^{k}\right)=c_{k+1}-s_{k+1}-c_{k} \kappa_{k}+s_{k}=0$, and thus $c y^{k}-x^{k}=b$ for $k=1, \ldots, n$. Finally $(c,-1)\left(z^{1}-z^{0}\right)=c_{1}-s_{1}>0$. Thus $c y^{0}-x^{0}=c y^{0}<c y^{1}-x^{1}=b$ and $z^{0}$ is in $\mathcal{Q}_{\leq}$.

Observation 2 The $(\{1\}, \cdots,\{n\}) \leq$-partition inequality

$$
\sum_{j=1}^{n} \pi_{j} y_{j} \leq \pi_{0}+x
$$

passes through the $n+1$ points given in Observation 1 , where $\pi_{1}=s_{1}, \pi_{j}=$ $\kappa_{j-1} \pi_{j-1}+\left(s_{j}-s_{j-1}\right)$ for $j=2, \ldots, n$ and $\pi_{0}=\kappa_{n} \pi_{n}-s_{n}$. These points define the inequality uniquely (to within scalar multiplication) and are affinely independent.

Proof Let $e_{j}^{\prime}$ be the $j$ th unit vector of size $n+1$. We suppose that all the points satisfy the inequality $\sum_{j=1}^{n} \mu_{j} y_{j}-x \leq \mu_{0}$ at equality.

Since $z^{1}-z^{0}=e_{1}^{\prime}+s_{1} e_{n+1}^{\prime}$, we have $\mu_{1}=s_{1}$. For $j=2, \ldots, n, z^{j}-z^{j-1}=$ $e_{j}^{\prime}-\kappa_{j-1} e_{j-1}^{\prime}+\left(s_{j}-s_{j-1}\right) e_{n+1}^{\prime}$ and thus $\mu_{j}=\kappa_{j-1} \mu_{j-1}+\left(s_{j}-s_{j-1}\right)$. Finally $\mu y^{n}=\mu_{0}+s_{n}$ implies $\mu_{0}=\kappa_{n} \mu_{n}-s_{n}$. As the inequality is uniquely defined by the $n+1$ points, the points are affinely independent.

Observation 3 Let $\sum_{j=1}^{n} \alpha_{j} y_{j} \geq \alpha_{0}$ denote the $(\{1\},\{2\}, \cdots,\{n\})$ partition inequality (1) for the $\geq$-knapsack set $\mathcal{C}$. Then the $\leq$-partition inequality (3) for $\mathcal{Q}_{\leq}$can also be viewed as a lifting of this inequality, and can be written in the form

$$
\sum_{j=1}^{n}\left(c_{j}-\left(c_{1}-s_{1}\right) \alpha_{j}\right) y_{j} \leq\left(b-\left(c_{1}-s_{1}\right) \alpha_{0}\right)+x .
$$

Proof By Observation 2, the points $z^{k}$ satisfy (3) at equality. In addition $y^{0}=y^{1}-e_{1}$, so $c y^{0}=c y^{1}-c_{1}=b+s_{1}-c_{1}$ and $\alpha y^{0}=\alpha y^{1}-\alpha_{1}=\alpha_{0}-1$. So $z^{0}$ also lies on the proposed inequality and the inequalities must be identical.

\subsection{Validity of partition inequalities}

We need a refinement of the notation in this subsection. We denote the continuous $\leq$-knapsack set with $n$ integer variables by $\mathcal{Q}_{\leq}^{n}$ and the set with the first $n-1$ integer variables by $\mathcal{Q}_{\leq}^{n-1}$. Similarly $\pi_{0}^{n}$ is the right hand side of the partition inequality for 
$\mathcal{Q}_{\leq}^{n}$ and $\pi_{0}^{n-1}$ for $\mathcal{Q}_{\leq}^{n-1}$. In particular we will use validity of $\sum_{j=1}^{n-1} \pi_{j} y_{j} \leq \pi_{0}^{n-1}+x$ for $\mathcal{Q}_{\leq}^{n-1}$ to show the validity of $\sum_{j=1}^{n} \pi_{j} y_{j} \leq \pi_{0}^{n}+x$ for $\mathcal{Q}_{\leq}^{n}$.

We first establish some basic properties. We still assume that $c_{1}$ does not divide $b$. First note that

$$
\beta_{j}=b-\left\lfloor\frac{b}{c_{j+1}}\right\rfloor c_{j+1} \text { for } j=1, \ldots, n-1
$$

and

$$
s_{j}-s_{j-1}=\left\lfloor\frac{s_{j}}{c_{j-1}}\right\rfloor c_{j-1} \text { for } j=2, \ldots, n
$$

Lemma 1 i. $\pi_{j} \leq c_{j}$ for $j=1, \ldots, n$,

ii. $\frac{\pi_{j}}{c_{j}} \geq \frac{\pi_{j-1}}{c_{j-1}}$ for $j=2, \ldots, n$,

iii. $\pi_{0}^{n}=\pi_{0}^{n-1}+\left(\kappa_{n}-1\right)\left(\pi_{n}-\pi_{n-1} \frac{c_{n}}{c_{n-1}}\right)$,

iv. $\pi_{0}^{n}=b-\left(c_{n}-\pi_{n}\right) \kappa_{n}$.

Proof We use induction to prove part (i). For $j=1$, we have $\pi_{1}=s_{1} \leq c_{1}$. Suppose that $\pi_{j-1} \leq c_{j-1}$. Then $\pi_{j}=\pi_{j-1} \kappa_{j-1}+s_{j}-s_{j-1} \leq c_{j-1} \kappa_{j-1}+s_{j}-s_{j-1}$. The right hand side is equal to $c_{j-1}\left\lceil\frac{b-\left\lfloor\frac{b}{c_{j}}\right\rfloor c_{j}}{c_{j-1}}\right\rceil+c_{j}-c_{j-1}+\left\lfloor\frac{b}{c_{j}}\right\rfloor c_{j}-\left\lfloor\frac{b}{c_{j-1}}\right\rfloor c_{j-1}=c_{j}$. Hence $\pi_{j} \leq c_{j}$.

To prove (ii),

$$
\begin{aligned}
\pi_{j} & =\kappa_{j-1} \pi_{j-1}+s_{j}-s_{j-1} \\
& =\left\lceil\frac{c_{j}-s_{j}}{c_{j-1}}\right\rceil \pi_{j-1}+s_{j}-s_{j-1} \\
& =\frac{c_{j}}{c_{j-1}} \pi_{j-1}-\left\lfloor\frac{s_{j}}{c_{j-1}}\right\rfloor \pi_{j-1}+s_{j}-s_{j-1} \\
& \geq \frac{c_{j}}{c_{j-1}} \pi_{j-1}-\left\lfloor\frac{s_{j}}{c_{j-1}}\right\rfloor c_{j-1}+s_{j}-s_{j-1}=\frac{c_{j}}{c_{j-1}} \pi_{j-1},
\end{aligned}
$$

where the inequality is obtained using (i) and the last equality is obtained using $s_{j}-$ $s_{j-1}=\left\lfloor\frac{s_{j}}{c_{j-1}}\right\rfloor c_{j-1}$.

Next we prove part (iii). First, $\pi_{0}^{n}=\pi_{n} \kappa_{n}-s_{n}$ and

$$
\pi_{0}^{n-1}=\pi_{n-1}\left\lceil\frac{b}{c_{n-1}}\right\rceil-s_{n-1}=\pi_{n-1}\left(\kappa_{n-1}+\left(\kappa_{n}-1\right) \frac{c_{n}}{c_{n-1}}\right)-s_{n-1} .
$$

Now 


$$
\begin{aligned}
\pi_{0}^{n}-\pi_{0}^{n-1} & =\left(\kappa_{n}-1\right)\left(\pi_{n}-\pi_{n-1} \frac{c_{n}}{c_{n-1}}\right)+\pi_{n}-\pi_{n-1} \kappa_{n-1}-s_{n}+s_{n-1} \\
& =\left(\kappa_{n}-1\right)\left(\pi_{n}-\pi_{n-1} \frac{c_{n}}{c_{n-1}}\right)
\end{aligned}
$$

using the definition of $\pi_{n}$.

Finally, since $\pi_{0}^{n}=\kappa_{n} \pi_{n}-s_{n}$ and $s_{n}=c_{n}-\beta_{n-1}=\kappa_{n} c_{n}-b$, we have $\pi_{0}^{n}=$ $b-\left(c_{n}-\pi_{n}\right) \kappa_{n}$, which proves part (iv).

Lemma 2 If $\sum_{j=1}^{n-1} \pi_{j} y_{j} \leq \pi_{0}^{n-1}+x$ is valid for $\mathcal{Q}_{\leq}^{n-1}$, then

$$
\sum_{j=1}^{n-1} \pi_{j} y_{j}+\pi_{n-1} \frac{c_{n}}{c_{n-1}} y_{n} \leq \pi_{0}^{n-1}+x
$$

is valid for $\mathcal{Q}_{\leq}^{n}$.

Proof If $\left(y_{1}, \ldots, y_{n}, x\right) \in \mathcal{Q}_{\leq}^{n}$, then $\sum_{j=1}^{n} c_{j} y_{j}=\sum_{j=1}^{n-2} c_{j} y_{j}+c_{n-1}\left(y_{n-1}+\right.$ $\left.\frac{c_{n}}{c_{n-1}} y_{n}\right) \leq b+x$. Hence $\left(y_{1}, \ldots, y_{n-2}, y_{n-1}+\frac{c_{n}}{c_{n-1}} y_{n}, x\right) \in \mathcal{Q}_{\leq}^{n-1}$ and $\sum_{j=1}^{n-2} \pi_{j} y_{j}+$ $\pi_{n-1}\left(y_{n-1}+\frac{c_{n}}{c_{n-1}} y_{n}\right) \leq \pi_{0}^{n-1}+x$ as claimed.

Theorem 5 The $(\{1\}, \cdots,\{n\})$ partition inequality is valid for $\mathcal{Q}_{\leq}^{n}$.

Proof The proof is by induction.

For $n=1$, the MIR inequality is:

$$
c_{1}(1-f) y_{1} \leq\left\lfloor\frac{b}{c_{1}}\right\rfloor c_{1}(1-f)+x,
$$

where $(1-f)=\left\lceil\frac{b}{c_{1}}\right\rceil-\frac{b}{c_{1}}=\frac{s_{1}}{c_{1}}$. This is precisely the $(\{1\})$ partition inequality $s_{1} y_{1} \leq\left(\kappa_{1} s_{1}-s_{1}\right)+x$.

Now suppose that $\sum_{j=1}^{n-1} \pi_{j} y_{j} \leq \pi_{0}^{n-1}+x$ is valid for $\mathcal{Q}_{\leq}^{n-1}$. We consider two cases:

Case $1 y_{n} \geq \kappa_{n}$.

Suppose that $(y, x) \in \mathcal{Q}_{\leq}^{n}$, so that $\sum_{j=1}^{n} c_{j} y_{j} \leq b+x$, or rewriting $\sum_{j=1}^{n-1} c_{j} y_{j}+$ $c_{n}\left(y_{n}-\kappa_{n}\right)+c_{n} \kappa_{n} \leq b+x$. As $c_{j} \geq \pi_{j}$ by Lemma 1(i), $y_{j} \geq 0$ for $j=1, \ldots, n-1$ and $y_{n}-\kappa_{n} \geq 0$, we have $\sum_{j=1}^{n-1} \pi_{j} y_{j}+\pi_{n}\left(y_{n}-\kappa_{n}\right)+c_{n} \kappa_{n} \leq b+x$, or equivalently $\sum_{j=1}^{n-1} \pi_{j} y_{j}+\pi_{n} y_{n} \leq b-\left(c_{n}-\pi_{n}\right) \kappa_{n}+x$. Using Lemma 1(iv), we obtain $\sum_{j=1}^{n} \pi_{j} y_{j} \leq \pi_{0}^{n}+x$.

Case 2. $y_{n} \leq \kappa_{n}-1$.

From Lemma 2, $(y, x)$ satisfies $\sum_{j=1}^{n-1} \pi_{j} y_{j}+\pi_{n-1} \frac{c_{n}}{c_{n-1}} y_{n} \leq \pi_{0}^{n-1}+x$. Adding $\pi_{n}-\pi_{n-1} \frac{c_{n}}{c_{n-1}} \geq 0$ (from Lemma 1(ii)) times $y_{n} \leq \kappa_{n}-1$ gives $\sum_{j=1}^{n} \pi_{j} y_{j} \leq$ $\pi_{0}^{n-1}+\left(\kappa_{n}-1\right)\left(\pi_{n}-\pi_{n-1} \frac{c_{n}}{c_{n-1}}\right)+x=\pi_{0}^{n}+x$ where the last equality is obtained using Lemma 1(iii).

Therefore by a disjunctive argument, the inequality is valid for $\mathcal{Q}_{\leq}^{n}$. 
Example 1 Consider the continuous $\leq$-knapsack set defined by the constraints

$$
5 y_{1}+10 y_{2}+30 y_{3} \leq 72+x, y \in \mathbb{Z}_{+}^{3}, x \in \mathbb{R}_{+}^{1} .
$$

The coefficients of the $(\{1\}\{2\}\{3\})$ partition inequality are calculated as follows:

\begin{tabular}{rrrrr}
\hline$t$ & $\beta$ & $\kappa$ & $\mu$ & $s$ \\
\hline 3 & 72 & 3 & 60 & 18 \\
2 & 12 & 2 & 10 & 8 \\
1 & 2 & 1 & 0 & 3 \\
\hline
\end{tabular}

Then $\pi_{1}=3, \pi_{2}=1 \times 3+(8-3)=8, \pi_{3}=8 \times 2+(18-8)=26$ and $\pi_{0}=26 \times 3-18=60$ giving the inequality

$$
3 y_{1}+8 y_{2}+26 y_{3} \leq 60+x .
$$

Note that the $(\{1\}\{2\}\{3\}) \geq$-partition inequality for

$$
5 y_{1}+10 y_{2}+30 y_{3} \geq 72, \quad y \in \mathbb{Z}_{+}^{3}
$$

is the inequality

$$
y_{1}+y_{2}+2 y_{3} \geq 6 \text {. }
$$

Now $5 y_{1}+10 y_{2}+30 y_{3} \leq 72+x$ plus $c_{1}-s_{1}=2$ times the latter inequality again gives $3 y_{1}+8 y_{2}+26 y_{3} \leq 60+x$.

Now we describe the inequality associated with an arbitrary partition and we drop the assumption that $c_{1}$ does not divide $b$. Thus we suppose that $r$ is the unique index with $c_{r-1} \mid b$, but $c_{r} \not \supset b$. Note that $r \leq v(b)+1$ and $r=1$ implies that $c_{i} \not \supset b$ for all $i$.

Proposition 2 For the partition $\left\{i_{1}, \ldots, j_{1}\right\}, \ldots,\left\{i_{p}, \ldots, j_{p}\right\}$ of $\{r, \ldots, n\}$, the partition inequality

$$
\sum_{t=1}^{p} \pi_{i_{t}} \sum_{j=i_{t}}^{j_{t}} \frac{c_{j}}{c_{i_{t}}} y_{j} \leq \pi_{0}+x .
$$

is valid for $\mathcal{Q}_{\leq}$.

The set of points of $\mathcal{Q}_{\leq}$that satisfy (4) at equality is the union of the sets

$$
\begin{aligned}
Z^{k}= & \left\{(y, x) \in \mathbb{Z}_{+}^{n} \times \mathbb{R}^{1}: y_{j}=0 j<i_{k}, \sum_{j=i_{k}}^{j_{k}} \frac{c_{j}}{c_{i_{k}}} y_{j}=\kappa_{k},\right. \\
& \left.\sum_{j=i_{t}}^{j_{t}} \frac{c_{j}}{c_{i_{t}}} y_{j}=\kappa_{t}-1 t=k+1, \ldots, p, x=s_{k}\right\}
\end{aligned}
$$


for all $k=1, \ldots, p$ and

$Z^{0}=\left\{(y, x) \in \mathbb{Z}_{+}^{n} \times \mathbb{R}^{1}: \sum_{j=1}^{r-1} c_{j} y_{j} \leq \delta, \sum_{j=i_{t}}^{j_{t}} \frac{c_{j}}{c_{i_{t}}} y_{j}=\kappa_{t}-1 t=1, \ldots, p, x=0\right\}$

where $\delta=b-\sum_{t=1}^{p} c_{i_{t}}\left(\kappa_{t}-1\right)$.

Proof First observe that inequality $\sum_{t=1}^{p} \pi_{i_{t}} y_{i_{t}} \leq \pi_{0}+x$ is valid for the set $\sum_{t=1}^{p} c_{i_{t}} y_{i_{t}} \leq b+x, \quad y \in \mathbb{Z}_{+}^{p}, \quad x \in \mathbb{R}_{+}^{1}$. Now, the proof that the inequality (4) is valid for $\sum_{j=r}^{n} c_{j} y_{j} \leq b+x, y \in \mathbb{Z}_{+}^{n-r+1}, x \in \mathbb{R}_{+}^{1}$ is as in Lemma 2. The structure of the tight points follows from that of the tight points $\left\{z^{k}\right\}_{k=0}^{p}$ in Observation 2.

4.2 Decomposition and extended formulation for $\mathcal{Q}_{\leq}$

Here we show how the set $\mathcal{Q}_{\leq}$can be decomposed allowing one to derive a polynomial size extended formulation for $\operatorname{conv}\left(\mathcal{Q}_{\leq}\right)$. First we look at the simple cases.

Observation 4 If $c_{1}|\cdots| c_{n} \mid b$, the polyhedron

$$
\left\{(y, x) \in \mathbb{R}_{+}^{n} \times \mathbb{R}_{+}^{1}: \sum_{j} c_{j} y_{j} \leq b+x\right\}
$$

is integral and describes $\operatorname{conv}\left(\mathcal{Q}_{\leq}\right)$.

Observation 5 If $c_{j} \mid b$ for $j=1, \ldots, v(b)$ and $v(b)<n$, then $\operatorname{conv}\left(\mathcal{Q}_{\leq}\right)$is described by the original constraints $c y \leq b+x, y, x \geq 0$ and one additional constraint

$$
\sum_{j=1}^{n}\left(c_{j}-b\right)^{+} y_{j} \leq x \text {. }
$$

\subsubsection{Decomposition of $\operatorname{conv}\left(\mathcal{Q}_{\leq}\right)$}

To avoid the case covered in Observation 5, we assume $r \leq v(b)$. Let

$$
\mathcal{U}=\left\{(y, x) \in \mathbb{Z}_{+}^{n} \times \mathbb{R}_{+}^{1}: \sum_{j=1}^{v(b)} c_{j} y_{j}+\sum_{j=v(b)+1}^{n}\left(c_{j}-K\right) y_{j} \leq b-K+x\right\}
$$

where $K=\left\lfloor\frac{b}{c_{v(b)}}\right\rfloor c_{v(b)}$.

Let $\mathcal{R}$ be the set of vectors $(y, x, \alpha, \gamma, \delta)$ that satisfy

$$
\begin{aligned}
& \sum_{j=1}^{v(b)} c_{j} \alpha_{j}+K \sum_{j=v(b)+1}^{n} \alpha_{j} \leq K \\
& \left(\gamma, \gamma_{0}\right) \in \operatorname{conv}(\mathcal{U})
\end{aligned}
$$




$$
\begin{array}{ll}
y_{j}=\alpha_{j}+\gamma_{j} & j=1, \ldots, v(b) \\
y_{j}=\gamma_{j}+\delta_{j}, \gamma_{j}=\alpha_{j} & j=v(b)+1, \ldots, n \\
x \geq \gamma_{0}+\sum_{j=v(b)+1}^{n} c_{j} \delta_{j} & \\
\alpha, \delta \in \mathbb{R}_{+}^{n} . &
\end{array}
$$

Proposition $3 \operatorname{proj}_{y, x} \mathcal{R}=\operatorname{conv}\left(\mathcal{Q}_{\leq}\right)$.

Proof From the first constraint defining $\mathcal{R}$, we see that $\alpha_{j}=0$ for all $j$ in any extreme ray of $\mathcal{R}$. The extreme rays $\left(e_{j}, c_{j}\right)$ for $j=1, \ldots, v(b)$ and $(0,1)$ of $\operatorname{conv}(\mathcal{U})$ also become extreme rays of $\operatorname{proj}_{y, x} \mathcal{R}$ by taking $\gamma_{j}=1$ and $\gamma_{0}=1$ respectively. The variables $\delta_{j}$ provide additional rays $\left(e_{j}, c_{j}\right)$ for $j>v(b)$. Thus the rays of the two sets are the same. In addition it is straightforward to check that the points $(0,0)$ and the maximal points for the partition inequalities given in Proposition 2 lie in $\operatorname{proj}_{y, x} \mathcal{R}$.

To show that $\operatorname{proj}_{y, x} \mathcal{R} \subseteq \operatorname{conv}\left(\mathcal{Q}_{\leq}\right)$, consider a point $(y, x) \in \operatorname{proj}_{y, x} \mathcal{R}$. Thus there exist $(\alpha, \gamma, \delta)$ such that $(y, x, \alpha, \gamma, \delta) \in \mathcal{R}$. Let $I$ be the set of extreme points of $\operatorname{conv}(\mathcal{U})$ with $\sum_{j=v(b)+1}^{n} \gamma_{j}=0$. It is straightforward to check that the only other extreme points are the points $\left(e_{j}, c_{j}-b\right)$ for $j=v(b)+1, \ldots, n$. As $\left(\gamma, \gamma_{0}\right) \in$ $\operatorname{conv}(\mathcal{U})$, we can write

$$
\begin{aligned}
\left(\gamma, \gamma_{0}\right)= & \sum_{i \in I}\left(\gamma^{i}, \gamma_{0}^{i}\right) \lambda_{i}+\sum_{j=v(b)+1}^{n}\left(e_{j}, c_{j}-b\right) \epsilon_{j} \\
& +\sum_{j=1}^{v(b)}\left(e_{j}, c_{j}\right) \delta_{j}+\sum_{j=v(b)+1}^{n}\left(e_{j}, c_{j}-K\right) \phi_{j}+(0,1) \phi_{0}
\end{aligned}
$$

where $\sum_{i \in I} \lambda_{i}+\sum_{j=v(b)+1}^{n} \epsilon_{j}=1, \lambda, \epsilon, \phi, \delta \geq 0$.

Also

$$
\alpha=\sum_{j=0}^{n} \alpha^{j} v_{j}, \sum_{j=0}^{n} v_{j}=1, v \geq 0
$$

where $\alpha^{j}$ for $j=0, \ldots, n$ are the extreme points of $\left\{\alpha \in \mathbb{R}_{+}^{n}: \sum_{j=1}^{v(b)} c_{j} \alpha_{j}\right.$ $\left.+K \sum_{j=v(b)+1}^{n} \alpha_{j} \leq K\right\}$. Specifically $\alpha^{0}=0, \alpha^{j}=\frac{K}{c_{j}} e_{j}$ for $j=1, \ldots, v(b)$ and $\alpha^{j}=e_{j}$ for $j=v(b)+1, \ldots, n$.

Then for $(y, x) \in \operatorname{proj}_{y, x} \mathcal{R}$, we have

$$
\begin{aligned}
(y, x)= & \sum_{i \in I}\left(\gamma^{i}, \gamma_{0}^{i}\right) \lambda_{i}+\sum_{j=0}^{v(b)}\left(\alpha^{j}, 0\right) \nu_{j}+\sum_{j=v(b)+1}^{n}\left(e_{j}, c_{j}-b\right) \epsilon_{j} \\
& +\sum_{j=v(b)+1}^{n}\left(e_{j}, c_{j}-K\right) \phi_{j}+\sum_{j=1}^{n}\left(e_{j}, c_{j}\right) \delta_{j}+(0,1) \phi_{0} .
\end{aligned}
$$


Let $\rho=\sum_{i \in I} \lambda_{i}$ and $\sigma=\sum_{j=0}^{v(b)} v_{j}$. For $j=v(b)+1, \ldots, n$, since $\gamma_{j}=\alpha_{j}$, we have $\epsilon_{j}+\phi_{j}=v_{j}$. Also $\sum_{j=v(b)+1}^{n} \phi_{j}=\sum_{j=v(b)+1}^{n}\left(v_{j}-\epsilon_{j}\right)=1-\sigma-(1-$ $\left.\sum_{i \in I} \lambda_{i}\right)=\rho-\sigma$.

Let $\left(y^{i j}, y_{0}^{i j}\right)=\left(\gamma^{i}, \gamma_{0}^{i}\right)+\left(\alpha^{j}, 0\right)$ for $i \in I$ and $j=0, \ldots, v(b)$. Clearly $\left(y^{i j}, y_{0}^{i j}\right) \in \mathcal{Q}_{\leq}$. Also let $\left(z^{i j}, z_{0}^{i j}\right)=\left(\gamma^{i}, \gamma_{0}^{i}\right)+\left(e_{j}, c_{j}-K\right)$ for $i \in I$ and $j=v(b)+1, \ldots, n$. As $c \gamma^{i} \leq b-K+\gamma_{0}^{i}$, we have $c z^{i j}=c \gamma^{i}+c_{j} \leq b+\left(\gamma_{0}^{i}+c_{j}-K\right)$ and thus $\left(z^{i j}, z_{0}^{i j}\right) \in \mathcal{Q}_{\leq}$. Also $\left(e_{j}, c_{j}-b\right) \in \mathcal{Q}_{\leq}$for $j>v(b)$.

Now since

$$
\begin{aligned}
& \frac{\sigma}{\rho} \sum_{i \in I}\left(\gamma^{i}, \gamma_{0}^{i}\right) \lambda_{i}+\sum_{j=0}^{v(b)}\left(\alpha^{j}, 0\right) v_{j}=\sum_{i \in I} \sum_{j=0}^{v(b)}\left(\left(\gamma^{i}, \gamma_{0}^{i}\right)+\left(\alpha^{j}, 0\right)\right) \frac{1}{\rho} \lambda_{i} v_{j} \\
& \quad=\sum_{i \in I} \sum_{j=0}^{v(b)}\left(y^{i j}, y_{0}^{i j}\right) \frac{1}{\rho} \lambda_{i} v_{j}
\end{aligned}
$$

and

$$
\begin{aligned}
& \frac{\rho-\sigma}{\rho} \sum_{i \in I}\left(\gamma^{i}, \gamma_{0}^{i}\right) \lambda_{i}+\sum_{j=v(b)+1}^{n}\left(e_{j}, c_{j}-K\right) \phi_{j} \\
& =\sum_{i \in I} \sum_{j=v(b)+1}^{n}\left(\left(\gamma^{i}, \gamma_{0}^{i}\right)+\left(e_{j}, c_{j}-K\right)\right) \frac{1}{\rho} \lambda_{i} \phi_{j} \\
& =\sum_{i \in I} \sum_{j=v(b)+1}^{n}\left(z^{i j}, z_{0}^{i j}\right) \frac{1}{\rho} \lambda_{i} \phi_{j},
\end{aligned}
$$

we can rewrite $(y, x)$ as a convex combination of extreme points plus extreme rays

$$
\begin{aligned}
(y, x)= & \left(\sum_{i \in I} \sum_{j=0}^{v(b)}\left(y^{i j}, y_{0}^{i j}\right) \frac{1}{\rho} \lambda_{i} v_{j}+\sum_{i \in I} \sum_{j=v(b)+1}^{n}\left(z^{i j}, z_{0}^{i j}\right) \frac{1}{\rho} \lambda_{i} \phi_{j}\right. \\
& \left.+\sum_{j=v(b)+1}^{n}\left(e_{j}, c_{j}-b\right) \epsilon_{j}\right) \\
& +\sum_{j=1}^{n}\left(e_{j}, c_{j}\right) \delta_{j}+(0,1) \phi_{0},
\end{aligned}
$$

as $\sum_{i \in I} \sum_{j=0}^{v(b)} \frac{1}{\rho} \lambda_{i} v_{j}+\sum_{i \in I} \sum_{j=v(b)+1}^{n} \frac{1}{\rho} \lambda_{i} \phi_{j}+\sum_{j=v(b)+1}^{n} \epsilon_{j}=\sigma+(\rho-\sigma)+$ $(1-\rho)=1$ and all multipliers are nonnegative. Thus $(y, x)$ lies in $\operatorname{conv}\left(\mathcal{Q}_{\leq}\right)$. 


\subsubsection{An extended formulation for $\operatorname{conv}\left(\mathcal{Q}_{\leq}\right)$}

As before, we assume that $c_{j} \mid b$ for $j<r, c_{r}$ does not divide $b, c_{v(b)}<b$ and $c_{j}>b$ for $j>v(b)$. Repeating the decomposition a maximum of $v(b)-r$ times, one terminates with a set $\mathcal{U}$ of the form

$$
\left\{(y, x) \in \mathbb{Z}_{+}^{n} \times \mathbb{R}_{+}^{1}: \sum_{j=1}^{r-1} c_{j} y_{j}+\sum_{j=r}^{n} \tilde{c}_{j} y_{j} \leq b-\left\lfloor\frac{b}{c_{r}}\right\rfloor c_{r}+x\right\}
$$

where $c_{j} \mid\left(b-\left\lfloor\frac{b}{c_{r}}\right\rfloor c_{r}\right)$ for $j<r$ and $\tilde{c}_{j}>\left(b-\left\lfloor\frac{b}{c_{r}}\right\rfloor c_{r}\right)$ for $j \geq r$, so Observation 5 gives $\operatorname{conv}(\mathcal{U})$ completing the polynomial size extended formulation.

Example 2 Consider a set $\mathcal{Q}_{\leq}$with $n=5, c=(3,6,18,90,180)$ and $b=737$.

With $r=1$ and $v(b)=5$, this decomposes into $3 \alpha_{11}+6 \alpha_{12}+18 \alpha_{13}+90 \alpha_{14}+$ $180 \alpha_{15} \leq 720$ and the convex hull of the set:

$$
\left\{\left(\gamma, \gamma_{0}\right) \in \mathbb{Z}_{+}^{5} \times \mathbb{R}_{+}^{1}: 3 \gamma_{1}+6 \gamma_{2}+18 \gamma_{3}+90 \gamma_{4}+180 \gamma_{5} \leq 17+\gamma_{0} \cdot\right\}
$$

The latter with $r=1$ and $v(17)=2$ decomposes into $3 \alpha_{21}+6 \alpha_{22}+12 \alpha_{23}+$ $12 \alpha_{24}+12 \alpha_{25} \leq 12$ and the convex hull of the set:

$$
\left\{\left(\gamma, \gamma_{0}\right) \in \mathbb{Z}_{+}^{5} \times \mathbb{R}_{+}^{1}: 3 \gamma_{1}+6 \gamma_{2}+(18-12) \gamma_{3}+78 \gamma_{4}+168 \gamma_{5} \leq 5+\gamma_{0}\right\}
$$

with $\gamma_{3}=\alpha_{23}, \gamma_{4}=\alpha_{24}$ and $\gamma_{5}=\alpha_{25}$.

The latter with $r=1$ and $v(5)=1$ decomposes into $3 \alpha_{31}+3 \alpha_{32}+3 \alpha_{23}+3 \alpha_{24}+$ $3 \alpha_{25} \leq 3$ and the convex hull of the set:

$$
\left\{\left(\gamma, \gamma_{0}\right) \in \mathbb{Z}_{+}^{5} \times \mathbb{R}_{+}^{1}: 3 \gamma_{1}+(6-3) \gamma_{2}+(6-3) \gamma_{3}+75 \gamma_{4}+165 \gamma_{5} \leq 2+\gamma_{0}\right\}
$$

with $\gamma_{2}=\alpha_{32}, \gamma_{3}=\alpha_{23}, \gamma_{4}=\alpha_{24}$ and $\gamma_{5}=\alpha_{25}$.

This is of the form treated in Observation 5, so to complete the convex hull of the latter, we add the constraints

$$
\begin{aligned}
3 \gamma_{1}+(6-3) \gamma_{2}+(6-3) \gamma_{3}+75 \gamma_{4}+165 \gamma_{5} & \leq 2+\gamma_{0} \\
(3-2) \gamma_{1}+1 \gamma_{2}+1 \gamma_{3}+73 \gamma_{4}+163 \gamma_{5} & \leq \gamma_{0} .
\end{aligned}
$$

with $\left(\gamma_{1}, \gamma_{2}, \gamma_{3}, \gamma_{4}, \gamma_{5}\right)=\left(\alpha_{41}, \alpha_{32}, \alpha_{23}, \alpha_{24}, \alpha_{25}\right)$.

The complete extended formulation is:

$$
\begin{aligned}
& 3 \alpha_{11}+6 \alpha_{12}+18 \alpha_{13}+90 \alpha_{14}+180 \alpha_{15} \leq 720 \\
& 3 \alpha_{21}+6 \alpha_{22}+12 \alpha_{23}+12 \alpha_{24}+12 \alpha_{25} \leq 12 \\
& 3 \alpha_{31}+3 \alpha_{32}+3 \alpha_{23}+3 \alpha_{24}+3 \alpha_{25} \leq 3 \\
& 3 \alpha_{41}+3 \alpha_{32}+3 \alpha_{23}+75 \alpha_{24}+165 \alpha_{25} \leq 2+\gamma_{0} . \\
& 1 \alpha_{41}+1 \alpha_{32}+1 \alpha_{23}+73 \alpha_{24}+163 \alpha_{25} \leq \gamma_{0}
\end{aligned}
$$




$$
\begin{aligned}
& y_{1}=\alpha_{11}+\alpha_{21}+\alpha_{31}+\alpha_{41}+\delta_{1} \\
& y_{2}=\alpha_{12}+\alpha_{22}+\alpha_{32}+\delta_{2} \\
& y_{3}=\alpha_{13}+\alpha_{23}+\delta_{3} \\
& y_{4}=\alpha_{14}+\alpha_{24}+\delta_{4} \\
& y_{5}=\alpha_{15}+\alpha_{25}+\delta_{5} \\
& x \geq \gamma_{0}+3 \delta_{1}+6 \delta_{2}+18 \delta_{3}+90 \delta_{4}+180 \delta_{5} \\
& \alpha_{i j} \geq 0 \forall i, j, \quad \delta \in \mathbb{R}_{+}^{5} .
\end{aligned}
$$

\section{The multi-item continuous $\leq-$ knapsack set}

Finally, we study the multi-item continuous $\leq$-knapsack set

$$
\mathcal{Y}_{\leq}=\left\{(y, x) \in \mathbb{Z}_{+}^{n} \times \mathbb{R}_{+}^{m+1}: \sum_{j \in N} c_{j} y_{j} \leq b+\sum_{i \in M_{0}} x_{i}, x_{i} \leq a_{i}, i \in M\right\} .
$$

Proposition 4 Let $S \subseteq M, B(S)=b+a(M \backslash S)$ and $r(S)$ be the smallest index $j$ such that $c_{j}$ does not divide $B(S)$. Let $q \in\{r(S), \ldots, n\}$ and $\left\{i_{1}, \ldots, j_{1}\right\}, \cdots,\left\{i_{p}, \ldots, j_{p}\right\}$ be a partition of $\{q, \ldots, n\}$. Define $\beta_{p}=B(S), \kappa_{t}=\left\lceil\frac{\beta_{t}}{c_{i_{t}}}\right\rceil, \mu_{t}=\left(\kappa_{t}-1\right) c_{i_{t}}$, $\beta_{t-1}=\beta_{t}-\mu_{t}$ and $s_{t}=c_{i_{t}}-\beta_{t-1}$ for $t=p, \ldots, 1$. Also let $\pi_{1}=s_{q}, \pi_{t}=$ $\kappa_{t-1} \pi_{t-1}+\left(s_{t}-s_{t-1}\right)$ for $t=2, \ldots, p$ and $\pi_{0}=\kappa_{p} \pi_{p}-s_{p}$. The partition inequality

$$
\sum_{t=1}^{p} \pi_{t} \sum_{j=i_{t}}^{j_{t}} \frac{c_{j}}{c_{i_{t}}} y_{j} \leq \pi_{0}+\sum_{i \in S_{0}} x_{i}
$$

is valid for $\mathcal{Y}_{\leq}$.

Proof Let

$$
\mathcal{Q}_{\leq}^{S}=\left\{(y, x) \in \mathbb{Z}_{+}^{n} \times \mathbb{R}^{m+1}: c y \leq B(S)+x\left(S_{0}\right), x\left(S_{0}\right) \geq 0\right\}
$$

By Proposition 2, we know that the partition inequality is valid for the set $\mathcal{Q}_{\leq}^{S}$ and this set is a relaxation of $\mathcal{Y}_{\leq}$.

Theorem $6 \operatorname{conv}\left(\mathcal{Y}_{\leq}\right)$is described by the initial constraints and the partition inequalities (5).

Proof Let $\Omega(g, h)$ be the set of optimal solutions to the problem of maximizing $\sum_{j=1}^{n} g_{j} y_{j}-\sum_{i=0}^{m} h_{i} x_{i}$ over $\mathcal{Y}_{\leq}$with $(g, h) \neq 0$.

If $h_{0}<0$ or if there exists $j \in N$ with $g_{j}>c_{j} h_{0}$, then the problem is unbounded. If $h_{i}<0$ for some $i \in M$, then all optimal solutions satisfy $x_{i}=a_{i}$. If $g_{j}<0$ or if there exists $i<j$ with $\frac{c_{j}}{c_{i}} g_{i}>g_{j}$, then $\Omega(g, h) \subseteq\left\{(y, x): y_{j}=0\right\}$.

Now suppose that $h_{0} \geq \frac{g_{n}}{c_{n}} \geq \ldots \geq \frac{g_{1}}{c_{1}} \geq 0$ and $h \geq 0$. If $h_{0}=0$ then $g=0$ and $\Omega(g, h) \subseteq\left\{(y, x): x_{i}=0\right\}$ for $i \in M$ with $h_{i}>0$. 
In the remaining case, we have $h_{0}>0, h \geq 0$ and $h_{0} \geq \frac{g_{n}}{c_{n}} \geq \ldots \geq \frac{g_{1}}{c_{1}} \geq 0$. Let $S=\left\{i \in M: h_{i}>0\right\}$. Note that if $(y, x)$ is an optimal solution with $c y<b+x\left(M_{0}\right)$, then $x\left(S_{0}\right)=0$, since otherwise one can decrease some $x_{i}$ with $i \in S_{0}$ by a small amount and improve the objective function value. Since $x\left(S_{0}\right)=0$, we also have $c y<b+x\left(M_{0} \backslash S_{0}\right) \leq b+a(M \backslash S)=B(S)$.

Consider the case in which there exists $j \in\{1, \ldots, r(S)-1\}$ with $g_{j}>0$. Take the smallest such $j$, so that $g_{i}=0$ for $i<j, g_{j}>0$ and $c_{j} \mid B(S)$. Suppose that there exists an optimal solution $(y, x)$ with $c y<b+x\left(M_{0}\right)$. One has $x\left(S_{0}\right)=0$ by optimality and setting $x_{i}=a_{i}$ for $i \in M \backslash S, y_{i}=0$ for all $i<j$ does not destroy feasibility or optimality. So $c y<B(S), c_{j} \mid B(S)$ and $c_{j} \mid c y$. Then $B(S)-c y$ is a positive multiple of $c_{j}, y_{j}$ can be increased by 1 , the resulting solution remains feasible and the objective value is increased, a contradiction. Thus $\Omega(g, h) \subseteq\left\{(y, x): c y=b+x\left(M_{0}\right)\right\}$.

From now on, we take $g_{j}=0$ for $j \in\{1, \ldots, r(S)-1\}$.

We look at three cases. In one case, $\Omega(g, h) \subseteq\left\{(y, x): x\left(S_{0}\right)=0\right\}$. In the second case, $\Omega(g, h) \subseteq\left\{(y, x): c y=b+x\left(M_{0}\right)\right\}$.

Finally in the third case, there exists an optimal solution $(y, x)$ with $x\left(S_{0}\right)>0$ and there exists an alternative optimal solution with $c y<b+x\left(M_{0}\right)$. Among the optimal solutions $(y, x)$ with $x\left(S_{0}\right)>0$, let $q$ be the smallest index for which there exists a solution with $y_{q}>0$. Note that $c y=B(S)+x\left(S_{0}\right)$ since $x\left(S_{0}\right)>0$. If $g_{q}=0$, one can decrease $y_{q}$ by 1 , decrease $x\left(S_{0}\right)$ and thereby improve the objective function. Thus $g_{q}>0$ and $q \geq r(S)$ (since $g_{j}=0$ for all $j=1, \ldots, r(S)-1$ ). Also if $q>r(S)$, $\frac{g_{q}}{c_{q}}>\frac{g_{q-1}}{c_{q-1}}$ as otherwise there would be an alternative optimal solution with $y_{q-1}>0$.

Define $\phi(\sigma)=\min \left\{\sum_{i \in S_{0}} h_{i} x_{i}: x\left(S_{0}\right) \geq \sigma-B(S), x \in \mathbb{R}_{+}^{\left|S_{0}\right|}, x_{i} \leq a_{i}, i \in S\right\}$. If $\sigma \leq B(S)$, then $\phi(\sigma)=0$. For $B(S)<\sigma, \phi(\sigma)$ is piecewise linear, strictly increasing and convex. Let $\left(y^{*}, x^{*}\right)$ be an an optimal solution with $x^{*}\left(S_{0}\right)>0$ and $y_{q}^{*}>0$. Then since $c y^{*}$ is divisible by $c_{q}$, we have $c y^{*} \geq\left\lceil\frac{B(S)}{c_{q}}\right\rceil c_{q}$. In addition, optimality of $\left(y^{*}, x^{*}\right)$ implies that $g_{q} \geq \phi\left(c y^{*}\right)-\phi\left(c\left(y^{*}-e_{q}\right)\right)$.

Suppose that $(y, x) \in \mathcal{Y}_{\leq}$and $c y<\left\lfloor\frac{B(S)}{c_{q}}\right\rfloor c_{q}$. Let $y^{\prime}=y+e_{q}$. Then $c y^{\prime}=$ $c y+c_{q}<\left\lfloor\frac{B(S)}{c_{q}}\right\rfloor c_{q}+c_{q}=\left\lceil\frac{B(S)}{c_{q}}\right\rceil c_{q}$ (since $c_{q}$ does not divide $B(S)$ ). Now as $c y^{*} \geq\left\lceil\frac{B(S)}{c_{q}}\right\rceil c_{q}$, we have $c y^{\prime}<c y^{*}$. Then $g_{q} \geq \phi\left(c y^{*}\right)-\phi\left(c\left(y^{*}-e_{q}\right)\right)>$ $\phi\left(c y^{\prime}\right)-\phi\left(c\left(y^{\prime}-e_{q}\right)\right)$ where the strict inequality follows from the form of the function $\phi$ and the fact that $c y^{*}>B(S)$. Hence $(y, x)$ cannot be optimal. As a result, every optimal solution $(y, x)$ satisfies $c y \geq\left\lfloor\frac{B(S)}{c_{q}}\right\rfloor c_{q}$.

Now consider the partition $\left\{i_{1}, \ldots, j_{1}\right\}, \ldots,\left\{i_{p}, \ldots, j_{p}\right\}$ of $\{q, \ldots, n\}$ with $\frac{g_{j}}{c_{j}}=$ $\frac{g_{i_{t}}}{c_{i_{t}}}$ for $t=1, \ldots, p$ and $j \in\left\{i_{t}, \ldots, j_{t}\right\}$ and $\frac{g_{i_{t}}}{c_{i_{t}}}>\frac{g_{i_{t-1}}}{c_{i_{t-1}}}$ for $t=2, \ldots, p$.

We first consider optimal solutions with $x\left(S_{0}\right)=0$ and then optimal solutions with $x\left(S_{0}\right)>0$. In an optimal solution with $x\left(S_{0}\right)=0$, we have $\sum_{j=q}^{n} c_{j} y_{j}=\left\lfloor\frac{B(S)}{c_{q}}\right\rfloor c_{q}$ as $\frac{g_{q}}{c_{q}}>\frac{g_{q-1}}{c_{q-1}}$ or else $q=r(S)$ and $g_{q}>0$, and $\sum_{j=1}^{q-1} c_{j} y_{j} \leq B(S)-\left\lfloor\frac{B(S)}{c_{q}}\right\rfloor c_{q}$. Since $\frac{g_{i_{t}}}{c_{i_{t}}}>\frac{g_{i_{t-1}}}{c_{i_{t-1}}}$ for $t=2, \ldots, p$, we have $\sum_{j=i_{t}}^{j_{t}} \frac{c_{j}}{c_{i_{t}}} y_{j}=\kappa_{t}-1$ for $t=1, \ldots, p$. This point belongs to set $Z^{0}$ of Proposition 2 and so lies on the partition inequality. 
Now we consider an optimal solution $(y, x)$ with $x\left(S_{0}\right)>0$. Suppose that there exists $j$ with $y_{j}>0$ and $c\left(y-e_{j}\right) \geq B(S)$. Then as $c\left(y-e_{j}\right)>c y^{\prime}$, we have $g_{j} \leq \phi\left(c\left(y^{\prime}+e_{j}\right)\right)-\phi\left(c y^{\prime}\right)<\phi(c y)-\phi\left(c\left(y-e_{j}\right)\right)$. This contradicts the optimality of $(y, x)$. Hence, in any optimal solution $(y, x)$ with $x\left(S_{0}\right)>0$, we have $c\left(y-e_{j}\right)<$ $B(S)$ for all $j$ with $y_{j}>0$. Then for $(y, x) \in \Omega(g, h)$ with $x\left(S_{0}\right)>0$, we have $\sum_{j=i_{k}}^{n} c_{j} y_{j}=\left\lceil\frac{B(S)}{c_{i_{k}}}\right\rceil c_{i_{k}}$ for some $k \in\{1, \ldots, p\}, y_{j}=0$ for $j \in\left\{1, \ldots, i_{k}-1\right\}$, $\sum_{j=i_{t}}^{j_{t}} \frac{c_{j}}{c_{i_{t}}} y_{j}=\kappa_{t}-1$ for $t=k+1, \ldots, p, \sum_{j=i_{k}}^{j_{k}} \frac{c_{j}}{c_{i_{k}}} y_{j}=\kappa_{k}$ and $x\left(S_{0}\right)=s_{k}$. This point is in $Z^{k}$ of Proposition 2 and so again lies on the partition inequality.

We obtain two immediate corollaries.

Corollary $2 \operatorname{conv}\left(\mathcal{Q}_{\leq}\right)$is described by its initial constraints and the partition inequalities (4).

\section{Corollary 3}

$$
\operatorname{conv}\left(\mathcal{Y}_{\leq}\right)=\cap_{S \subseteq M} \operatorname{conv}\left(\mathcal{Q}_{\leq}^{S}\right) \cap\left\{(y, x): x_{i} \leq a_{i} i \in M\right\} .
$$

\section{Conclusion}

In this paper, we have studied the polyhedra associated with knapsack sets with integer and continuous variables and divisible capacities.

In particular, we have studied the continuous $\geq$-knapsack set (equivalently the splittable flow arc set) with multiple capacities (facilities) and given a description of the convex hull when the capacities are divisible. We have shown that $\operatorname{conv}\left(\mathcal{Y}_{\geq}\right)=$ $\cap_{S \subseteq M} \operatorname{conv}\left(\mathcal{Q}_{\geq}^{S}\right) \cap\{(y, x): x \leq a\}$ where $\mathcal{Q}_{\geq}^{S}$ is a continuous $\geq$-knapsack set for each $S \subseteq M$. As a corollary it follows that, in any non-trivial facet-defining inequality for $\operatorname{conv}\left(\mathcal{Y}_{\geq}\right)$, the coefficients of the continuous variables all take the values 0 or 1 (after scaling).

Consider the optimization problem $\min \left\{\sum_{i \in M} h_{i} x_{i}+\sum_{j \in N} g_{j} y_{j}:(y, x) \in \mathcal{Y}_{\geq}\right\}$. If $h_{i}<0, x_{i}=a_{i}$ in every optimal solution and it suffices to solve a smaller problem. Thus we can assume that $0 \leq h_{1} \leq \ldots \leq h_{m}$. Now there exists an optimal solution $(y, x)$ with $x_{j}=a_{j}$ for $j<i$ and $x_{j}=0$ for $j>$ $i$ for some $i$. Thus it suffices to solve the $m$ problems $z_{i}=\sum_{j: j<i} h_{j} a_{j}+$ $\min \left\{h_{i} x_{i}+\sum_{j \in N} g_{j} y_{j}: x_{i}+c y \geq b-\sum_{j: j<i} a_{j}, 0 \leq x_{i} \leq a_{i}, y \in \mathbb{Z}_{+}^{n}\right\}$ and take the best solution. Each of these can be represented by a polynomial size linear program, so the optimization problem is in $\mathcal{P}$. Thus separation of $\operatorname{conv}\left(\mathcal{Y}_{\geq}\right)$is polynomial using the ellipsoid algorithm.

Though polynomial time combinatorial separation algorithms are known both for the partition inequalities for the integer $\geq$-knapsack set and the residual capacity inequalities for the single facility splittable flow arc set (see Pochet and Wolsey [16] and Atamtürk and Rajan [3], respectively), we do not know such an efficient combinatorial algorithm to separate the exponential family of partition inequalities (2).

We have shown in Theorem 6 that a result similar to that of Theorem 3 holds for the corresponding multi-item continuous $\leq$-knapsack set $\mathcal{Y}_{\leq}$with the same consequences 
for polynomial optimization and facet structure. It is natural to ask if similar results hold for other continuous knapsack sets with some special structure. Recently Dash et al. have shown that such results hold when there are just $n=2$ integer variables and arbitrary coefficients $c_{1}, c_{2}$. [4].

Kianfar [7] has shown how the partition inequalities for the integer $\geq$-knapsack set with divisible capacities can be viewed as a special case of $n$-step MIR inequalities and thus generalized. It seems likely that a similar approach can be taken for the $\leq$-knapsack set.

Acknowledgments This text presents research results of the Belgian Program on Interuniversity Poles of Attraction initiated by the Belgian State, Prime Minister's Office, Science Policy Programming. The scientific responsibility is assumed by the authors. The research of the first author was supported in part by the FIM-Institute for Mathematical Research, ETH Zurich. The research of the second author is supported by the Turkish Academy of Sciences.

\section{References}

1. Agra, A., Constantino, M.: Description of 2-integer continuous knapsack polyhedra. Discrete Optim. 3, 95-110 (2006)

2. Atamtürk, A.: On the facets of the mixed integer knapsack polyhedron. Math. Program. B 98, 145-175 (2003)

3. Atamtürk, A., Rajan, D.: On splittable and unsplittable flow capacitated network design arc-set polyhedra. Math. Program. 92, 315-333 (2002)

4. Dash, S., Günlük, O., Wolsey, L.A.: The continuous knapsack set. In: Core discussion paper DP2014/7, Core, Université catholique de Louvain, Louvain-la-Neuve, Belgium (2014)

5. Gupte, A.: Convex hulls of superincreasing knapsacks and lexicographic orderings. Optim. Online (2012)

6. Kellerer, H., Pferschy, U., Pisinger, D.: Knapsack Problems. Springer, Berlin (2004)

7. Kianfar, K.: On n-step MIR and partition inequalities for integer knapsack and single-node capacitated flow sets. Discrete Appl. Math. 160, 1567-1582 (2012)

8. Laurent, M., Sassano, A.: A characterization of knapsacks with the max-flow min-cut property. Oper. Res. Lett. 11, 105-110 (1992)

9. Lovász, L.: Graph theory and integer programming. Ann. Discrete Math. 4, 141-158 (1979)

10. Magnanti, T.L., Mirchandani, P., Vachani, R.: The convex hull of two core capacitated network design polyhedra. Math. Program. 60, 233-250 (1993)

11. Magnanti, T.L., Mirchandani, P., Vachani, R.: Modeling and solving the two-facility capacitated network loading problem. Oper. Res. 43, 142-157 (1995)

12. Marchand, H., Wolsey, L.A.: The $0-1$ knapsack problem with a single continuous variable. Math. Program. 85, 15-33 (1999)

13. Marcotte, O.: The cutting stock problem and integer rounding. Math. Program. 33, 82-92 (1985)

14. Martello, S., Toth, P.: Knapsack Problems: Algorithms and Computer Interpretations. WileyInterscience, New York (1990)

15. Pochet, Y., Weismantel, R.: The sequential knapsack polytope. SIAM J. Optim. 8, 248-264 (1998)

16. Pochet, Y., Wolsey, L.A.: Integer knapsack and flow covers with divisible coefficients: polyhedra, optimization and separation. Discrete Appl. Math. 59, 57-74 (1995)

17. Richard, J.-P.P., de Farias Jr, I.R.: Lifted inequalities for 0-1 mixed integer programming: basic theory and algorithms. Math. Program. B 98, 89-113 (2003) 